\title{
A MULTIVARIATE STATISTICAL MODEL FOR ADVANCED STORM SURGE ANALYSES IN THE NORTH SEA
}

\author{
Thomas Wahl ${ }^{1}$, Jürgen Jensen ${ }^{1}$ and Christoph Mudersbach ${ }^{1}$
}

\begin{abstract}
The knowledge of the characteristics of possible storm surges is essential to perform integrated risk analyses, e.g. based on the source-pathway-receptor concept, including the storm surge analyses (source), modeling failure mechanisms of the flood protection measures (pathway) and the quantification of potential losses (receptor). Focusing on the source part, a stochastic storm surge generator for the south-eastern North Sea is presented. The input data for the model are high resolution sea level observations from tide gauges during extreme events. Followed by the parameterization and fitting parametric distribution functions to the data sets, Monte-Carlo-Simulations allow the reconstruction of a large number of synthetic storm surge events. The latter serve as input data for the risk analyses and contribute to improving the overall results. The occurrence probabilities of the simulated extreme events are estimated based on multivariate statistics considering Copula functions, accounting for the structure of dependence overlooking the margins.
\end{abstract}

Keywords: integrated risk analyses, stochastic storm surge simulation, multivariate statistics, Copula model

\section{INTRODUCTION}

The joint research project XtremRisK (Oumeraci et al., 2009) brings together scientists from different universities and agencies to perform integrated risk analyses following the source-pathwayreceptor concept (SPR-Concept; Oumeraci, 2004). Figure 1 shows the different work packages which are addressed within the risk analyses. The source part (subproject 1 in the XtremRisK project) includes the storm surge analyses, whereas empirical studies as well as statistical analyses and also some numerical simulation runs are conducted. The pathway part (subproject 2) includes the dike and dune breach scenarios before potential losses (receptor part; subproject 3 ) are quantified. In a last step (subproject 4) the overall results are brought together and the integrated risk analyses are performed.

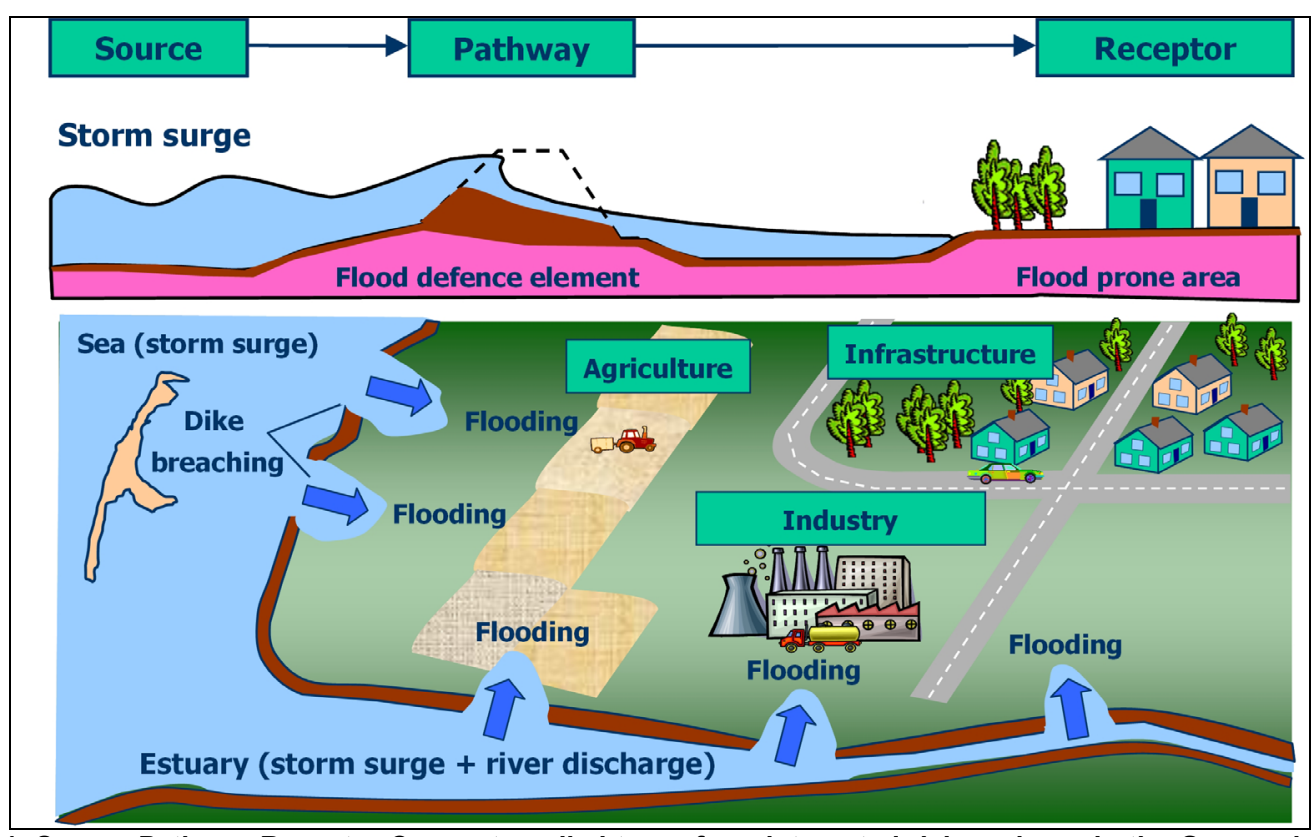

Figure 1. Source-Pathway-Receptor-Concept applied to perform integrated risk analyses in the German joint research project XtremRisK.

This contribution focuses on the source part and first of all on presenting the interim results from the statistical storm surge analyses. A stochastic storm surge generator is developed to allow the simulation of a large number of synthetic, physically consistent and high resolution storm surge events. Based on the results it is possible to estimate the occurrence probabilities of extreme storm surge events, which are an outcome of the empirical and numerical studies. As a significant improvement in

\footnotetext{
${ }^{1}$ Research Institute for Water and Environment, University of Siegen, Paul-Bonatz-Str. 9-11, 57076 Siegen
} 
comparison to former studies not only the highest water levels during storm surge events but also the storm surge intensities (also known as fullness) and thus implicitly the complete storm surge curves are considered for the statistical assessment. This implies that the results of the statistical analyses correspond to the following analyses comprising the modeling of failure mechanisms and the estimation of potential losses, where the complete storm surge curves are considered, too. In addition, the stochastically simulated storm surges may directly be considered for the risk analyses to improve the overall results by reducing the uncertainties.

The structure of the paper is as follows: In section 2 the investigation areas which are the island of Sylt and the city of Hamburg are introduced, the data basis for the subsequent analyses is presented and the problem of tide surge separation is briefly discussed. The most important steps stochastic storm surge simulation - storm surge parameterization, Monte-Carlo-Simulations and storm surge reconstruction - are presented in detail in Section 3. Section 4 focuses on the multivariate statistical assessment of the simulation results via 2-dimensional Archimedean Copula models. The paper concludes with a summary of the key findings and an outline for further research activities.

\section{INVESTIGATION AREAS, DATA BASIS AND THE PROBLEM OF TIDE SURGE SEPARATION}

Figure 2 shows the investigation area(s) and the locations of the tide gauges providing the data basis for the subsequent storm surge analyses. The island of Sylt was chosen to be one of the investigation areas as it hosts enormous values and counts among the most threatened parts of the German coast due to its exposed location. The tide gauge of Hörnum serves as 'reference tide gauge' for this investigation area. It is located in the south-east of the island and provides data from 1936 on, with only high and low waters available until 1999 and high resolution data (1-minute values) afterwards.

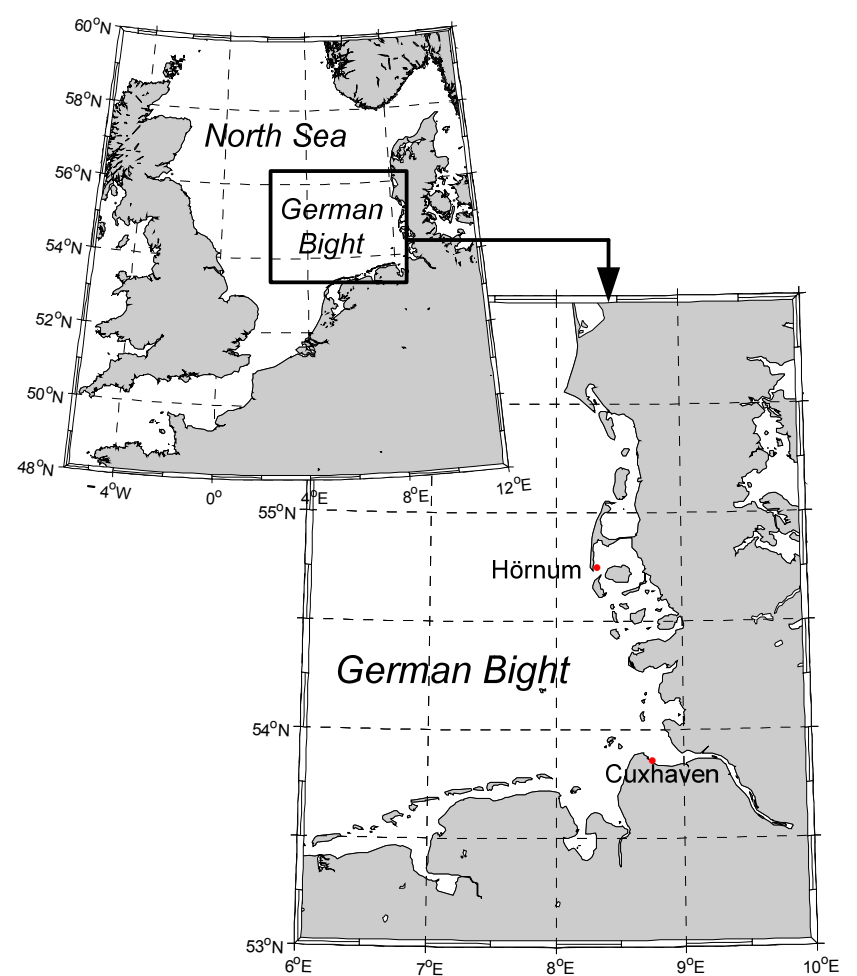

Figure 2. Investigation areas and locations of the tide gauges providing the data basis for the storm surge analyses.

The second investigation area is the megacity of Hamburg with about 1,8 million inhabitants and located at the Elbe estuary. The 'reference tide gauge' for the investigation area of Hamburg is the tide gauge of Cuxhaven, providing data from 1900 on (high and low waters until 1918 and hourly data afterwards). In this paper, we focus on the description of the applied methods and thus only the interim results for the investigation area of Sylt and from analyzing the tide gauge data of Hörnum are presented. 
At first, the extreme events observed at the tide gauge of Hörnum are identified based on the tidal high water time series. Here, a threshold of $1,40 \mathrm{~m}$ above mean high water is used to separate storm surge events from the observations, whereas two consecutive events have to be at least 30 hours apart from each other to approximately guarantee independency. Following these definitions, altogether 199 storm surge events are identified for the time period from 1936 to 2008 . For the following analyses high resolution water level time series of the identified extreme events are needed. Thus, the storm surge events observed between 1936 and 1999 were digitized within the project, whereas the tide curve with the storm surge peak water level and the two surrounding tides were considered. From analyzing the tidal high water time series, it was found that more than $99 \%$ of the observed storm surge events consisted of a maximum of three high tides in a row. As the main goal of our analyses consists in estimating occurrence probabilities of extreme storm surges, the consideration of three tides does not exclude longer storm surge events (for example consisting of five high tides in a row) from being analyzed in the project. It simply means that the occurrence probabilities of such long storm events are estimated based on three of the consisting tides, still taking much more information into account than in most former studies, where occurrence probabilities of storm surges were estimated solely based on the peak water levels (e.g. Jensen et al., 2006).

Furthermore, the above description points out that the observed water levels or tide curves, respectively, are analyzed and no tide surge separation is applied, as it might be recommended from a statistical point of view (Tawn 1988, 1992; Tawn and Dixon 1995, 1997). Also providing the possibility of making better use of the available data, there are different reasons (in our case) for not separating the stochastic component from the deterministic tidal signal before starting the analyses.

First of all tide surge separation supposes a certain degree of knowledge about the interaction between the two components in the investigation area and as joint probability methods are not implemented in German coastal design strategies up to now, the knowledge about the non-linear interaction processes is sparse. In addition, it is intended to stochastically simulate a large number of synthetic storm surges and when analyzing only the surge component it is required to re-combine both components in the end, which is hardly possible without detailed knowledge about the underlying nonlinear physical processes. Moreover, it has already been stated above that the main goal is the estimation of occurrence probabilities of extreme storm surge events and e.g. Tawn (1992) pointed out that a statistical analysis of a long time series consisting of annual high waters leads to similar results than the analysis of a short time series consisting of separated surge residuals and that the errors are in the same order, too. Here, we have more than 100 years (Cuxhaven) and more than 70 years (Hörnum) of observations available, which is a good data basis anyway.

As the empirical storm surge analyses conducted within the XtremRisK-project (Gönnert et al. 2010) focus on studying the non-linear interaction between tides and surge residuals in the German Bight, we expect more valuable information to be available at the end of the project. This, perhaps, will enable the development of a revised version of the presented stochastic model focusing on analyzing tides and surge residuals separately.

\section{STOCHASTIC STORM SURGE SIMULATION}

\subsection{Parameterization of observed storm surge curves}

The first and probably the most important step of stochastic storm surge simulation represents the parameterization of observed storm surge curves. From extensive tests and sensitivity studies it was found that a number of 19 sea level and 6 time parameters is sufficient to parameterize a typical storm surge event in the German Bight consisting of three tides. Figure 3 shows that the tidal high and low waters are used as sea level parameters as well as the observed water levels one hour before and hour afterwards. The six time parameters describe the time periods between two adjacent high and low waters.

The 25 parameters are estimated for all 199 observed storm surge events. Figure 4 shows the results for one single storm surge event, which has been observed at the tide gauge of Hörnum in January 2000. The red curve represents the observation, whereas the blue curve is the result from smoothing the observed curve (applying a pseudo-Gaussian low pass filter) to filter out high frequency variability and making it easier to automatically detect the selected sea level and time parameters (shown as black dots in Figure 4). 


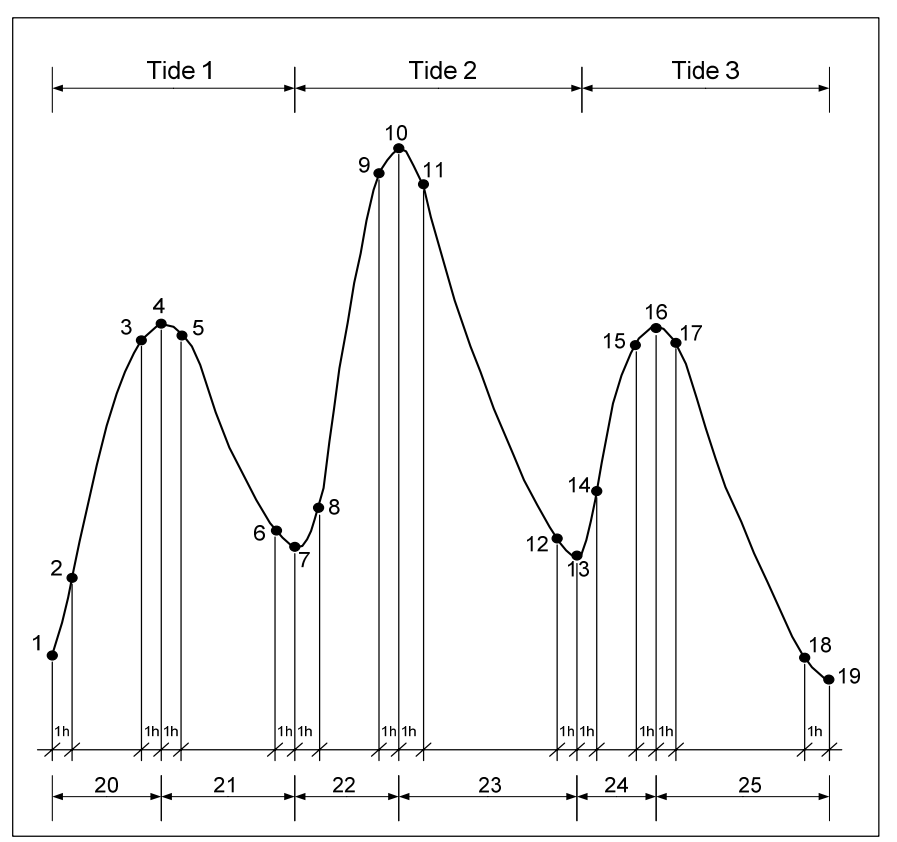

Figure 3. Parameters used to parameterize a typical storm surge event in the German Bight.

The green curve in Figure 4 represents the reconstructed storm surge curve, which is based solely on the 25 parameters. For the reconstruction we use Cubic Hermite interpolation (Kahaner et al. 1989; also known as Cubic Hermite spline or Cspline) which was found to be the most appropriate method leading to smaller mean squared errors (MSE) compared to the observed storm surge curves than other reconstruction methods, such as linear interpolation or cubic splines. For the example in Figure 4 the reconstruction results are very good, as the green curve is almost identical to the red or the blue curve, respectively. From visually checking the parameterization and reconstruction results for all 199 storm surge events it was found that similar results were achieved for the other events. Resulting from the parameterization, time series for all of the 25 parameters with each one consisting of 199 realizations are available.

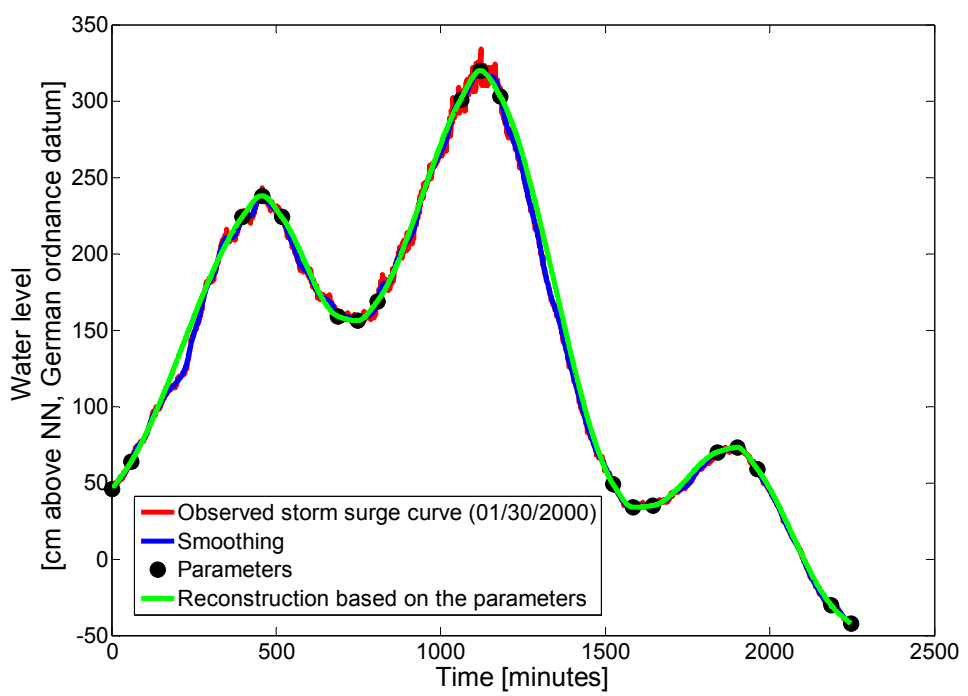

Figure 4. Results from parameterization and reconstruction of a storm surge event consisting of three tides.

\subsection{Monte-Carlo-Simulations}

In the next step a large number of Monte-Carlo-Simulations is conducted to create the data basis for the following reconstruction of synthetic storm surge events. Therefore, common parametric distribution functions (see first two columns of Table 1) are fitted to the data of the 25 time series 
resulting from the parameterization. The most suitable distribution for the particular data set is selected by estimating the MSE compared to the plotting positions (PLP), which are calculated by following the approach proposed by Gringorten (1963):

$$
P L P_{\text {Gringorten }}=\frac{m-0,44}{N+0,12}
$$

where $\mathrm{N}$ is the number of observations and $\mathrm{m}$ is the rank in the data vector sorted in ascending order. The distribution function providing the smallest MSE is evaluated and plotted against the PLP's to undergo an additional visual inspection. Figure 5 shows the results from fitting the distribution functions listed in Table 1 to the time series of the parameter 10, which is the time series consisting of the observed storm surge peak water levels (cf. Figure 3). As it could be expected from analyzing a time series of extreme events identified by a peak over threshold method, the Generalized Pareto distribution is found to provide the smallest MSE for this parameter.

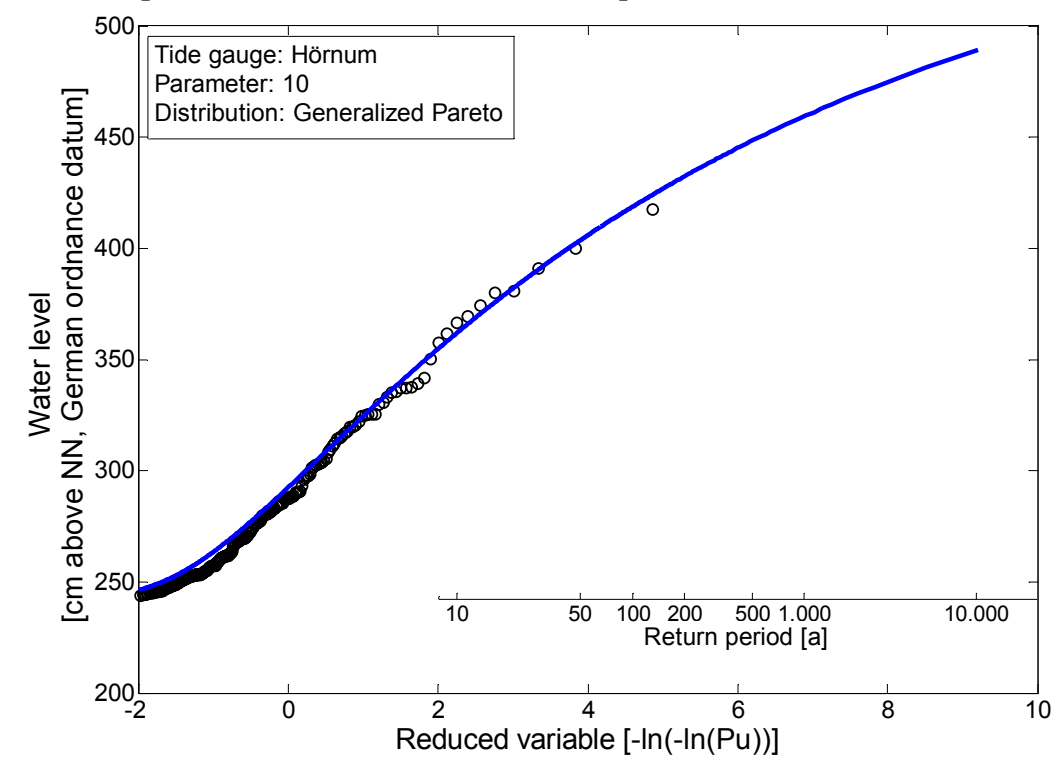

Figure 5. Results from fitting a Generalized Pareto Distribution to the time series of the peak water levels of the observed storm surge events (parameter 10).

As already mentioned, Table 1 shows an overview of the considered parametric distribution functions (columns one and two). The last column shows the overall results from fitting the distribution functions to the 25 time series of the 25 parameters. It can be seen, that the Generalized Pareto distribution provides the smallest MSE solely for the time series of parameter 10 and that a LogNormal, a Normal or a Weibull distribution provide smaller MSE`s for the rest of the parameters. This is due to the fact that only the highest water levels (parameters 10) are considered as absolute water levels, whereas the other parameters are relative water levels. The tidal high and low waters (parameters 1, 4, 7, 13, 16 and 19) are related to the highest turning point and the sea level parameters surrounding the high and low waters are related to the nearest turning point. The visual inspection of the results pointed out that a suitable distribution function could be found for each of the parameter time series from the pool of functions considered so far. The integration of additional parametric distribution functions is possible if required.

Dependencies between the parameters are considered by modeling the observed dependence through first order polynomial functions and assigning it to the simulation results before starting the reconstruction of synthetic storm surge events. It was found that dependencies are evident between the tidal high and low waters (parameters 1, 4, 7, 13, 16 and 19) and the peak water level (parameter 10). As an upper boundary condition the results of a former research project on extreme storm surges in the German Bight are considered. For more information see Jensen et al. (2006). For the investigation area of Sylt this means that storm surges with peak water levels $>489 \mathrm{cmNN}$ are not considered for reconstruction and the following statistical assessment. 
Table 1. Common parametric distribution functions considered to identify suitable functions for each of the 25 parameter time series.

\begin{tabular}{|c|c|c|}
\hline Distribution & Equation & Parameters \\
\hline $\begin{array}{l}\text { Generalized } \\
\text { Pareto }\end{array}$ & $G P(x, t)=\left(\frac{1}{b}\right)\left[1+k \frac{(x-\theta)}{b}\right]^{-1-\frac{1}{k}}$ & 10 \\
\hline LogNormal & $\log N(x, t)=\frac{1}{x \cdot b \sqrt{2 \pi}} e^{\frac{-(\ln x-a)^{2}}{2 b^{2}}}$ & $5,7,22,25$ \\
\hline Normal & $N(x, t)=\frac{1}{b \sqrt{2 \pi}} e^{\frac{-(x-a)^{2}}{2 b^{2}}}$ & $\begin{array}{c}1,12,13,17 \\
18,19,20,21 \\
23\end{array}$ \\
\hline Uniform & $U(x, t)=\frac{x-c}{d-c} I[c, d](x)$ & - \\
\hline Weibull & $W B L(x, t)=1-e^{-\left(\frac{x}{b}\right)^{k}} I[0, \infty](x)$ & $\begin{array}{c}2,3,4,6,8,9 \\
11,14,15,16 \\
24\end{array}$ \\
\hline
\end{tabular}

\subsection{Reconstruction and simulation results}

After running the Monte-Carlo-Simulations much more realizations for each of the 25 parameters are available and serve as a basis for the reconstruction of extreme storm surge events with a resolution in time of 1-minute. To display the results of the reconstruction of 1,000,000 (default number at the moment) synthetic storm surge events, the parameters 'highest turning point' (S) and 'intensity | fullness' (F) are used, which are also considered for the statistical assessment of the simulation results. Figure 6 shows that the storm surge intensity is defined as the area between the observed storm surge curve and Normal Null (NN, German ordnance datum), which is approximately the mean sea level height. Thus, the combined consideration of the two mentioned parameters gives an approximation of the load (in terms of the energy input) for the coastal protection measures.

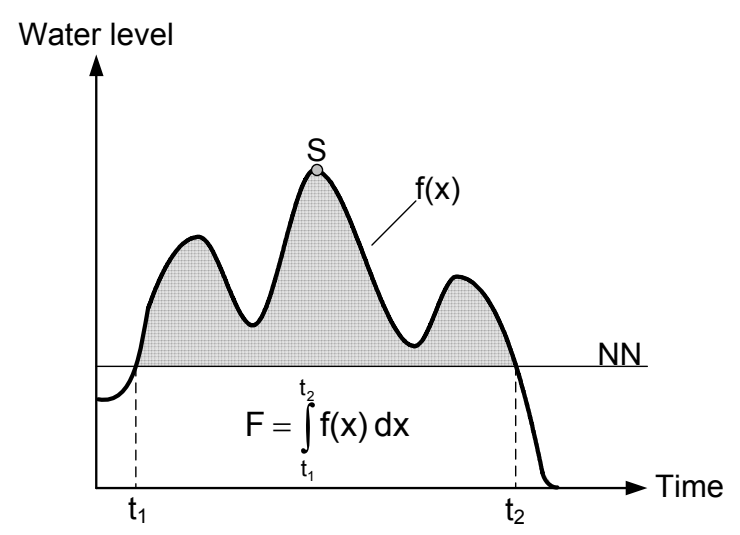

Figure 6. Definition of the parameter storm surge intensity (or fullness).

Figure 7 shows the results from simulating 1,000,000 synthetic storm surge curves, each one represented by the 'highest turning point' and the 'intensity | fullness'. The structure of dependence between the two parameters of the simulated storm surge events is the same compared to the observations and the simulation results involve all of the observed events.

In addition, the complete curves of three selected extreme events are plotted to point out, that each dot in the left hand figure represents such an event consisting of three tides and being available as a 
high resolution time series. To avoid implausible deformations of the simulated tide curves, some filter functions are applied after it has been proven that none of these filters influences the statistics.

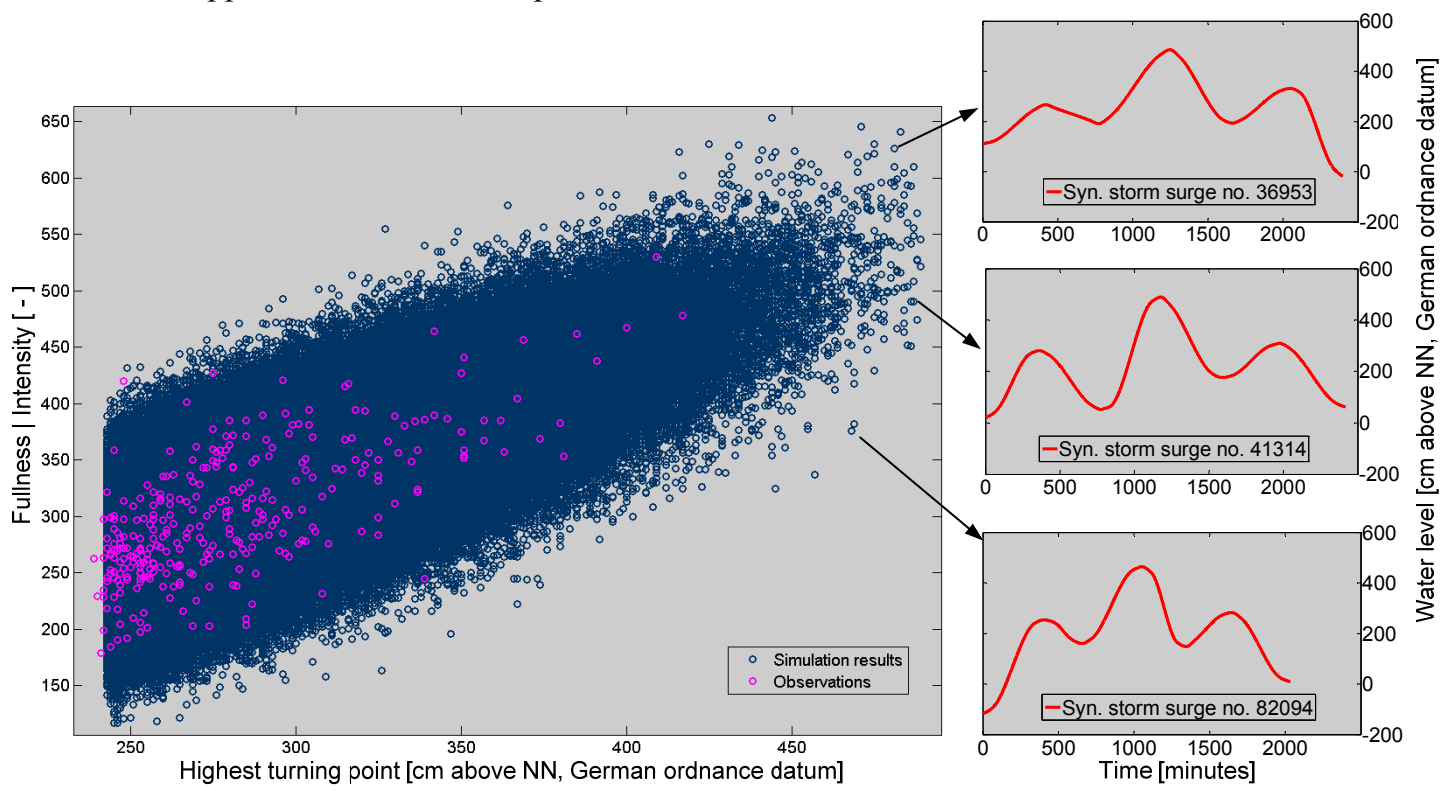

Figure 7. Overall results from stochastically simulating $1,000,000$ synthetic storm surge events (left) and the high resolution storm surge curves for three selected extreme events (right).

Based on the simulation results the statistical assessment of extreme events is possible and the resulting synthetic storm surges can additionally serve as the basis for a large number of investigations. Here, the simulated extreme events can be used for the risk analyses in combination with the results from the empirical and from numerical studies.

\section{MULTIVARIATE STATISTICAL ASSESSMENT}

From Figure 7 it can be seen that neither full dependency nor independency between the 'highest turning point' and the 'intensity | fullness' can be assumed. Thus, some bivariate model has to be applied to estimate the joint probabilities of the two parameters or the storm surge events, respectively. Therefore, in a first step some distribution functions have to be identified being suitable to describe the available realizations of the margins. From testing different parametric distribution functions it was found that a LogNormal distribution fits best (but not perfect) for the parameter 'intensity | fullness' and the parameter 'highest turning point' is best described by a Generalized Pareto distribution, which was considered for the Monte-Carlo-Simulations. In the literature, different bivariate statistical models are presented, such as the bivariate Gumbel model (Gumbel 1962) or the bivariate Gamma model (e.g. Yue et al. 2001). All of these models assume that both of the considered parameters can be adequately described by the same parametric distribution function (e.g. by a Gumbel distribution). But, as mentioned above it was found that the parameters considered here are differently distributed. Therefore, a 2-dimensional statistical model which can handle mixed marginal distributions has to be applied.

Copulas are flexible joint distributions that can be used to solve such multivariate statistical problems, where the margins provide some various dependency and follow different distribution functions (see e.g. Nelsen 1999). Copulas are widely used for financial risk assessments and actuarial analyses, but also in the field of hydrology, for example for inland flood risk assessments (e.g. Karmakar and Simonovic 2008, 2009; Klein et al. 2008). De Michele et al. (2007) analyze sea storms based on Copulas and de Waal and van Gelder (2005) apply Copulas to model extreme wave heights and periods. However, literature on the application of Copulas in the coastal engineering sector is sparse.

The advantage of having 1,000,000 realizations of the two storm surge parameters available and the fact that a further extrapolation of the marginal distributions is not necessary, can be used for the statistical assessment by considering non-parametric approaches, such as Kernel Density functions (Bowman and Azzalini 1997), as marginal distributions. Kernel density functions directly estimate the 
marginal univariate distribution function by smoothing the empirical frequency distribution of the underlying data set:

$$
K(x)=(2 \pi)^{-1 / 2} e^{-\left(x^{2}\right) / 2}, \text { with } \int_{-\infty}^{\infty} K(x) d x=1
$$

Applying Kernel Density functions reduces the uncertainties for the parameter 'intensity | fullness'. Figure 8 shows that discontinuities, which are evident for the most extreme events, are captured by the Kernel Density function, which is not the case with any parametric distribution function. For the parameter 'highest turning point', the results from considering a Kernel Density function or a Generalized Pareto distribution are the same as the Generalized Pareto distribution has been used for the Monte-Carlo-Simulations.

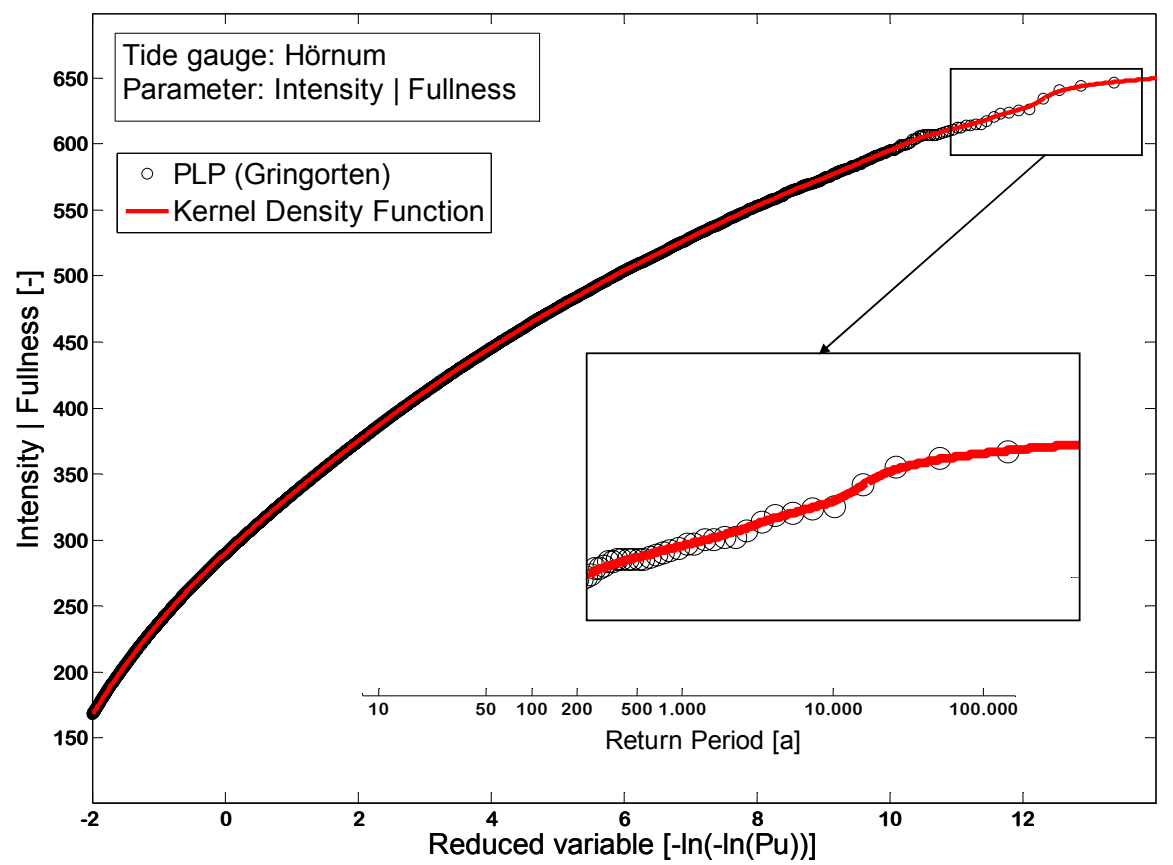

Figure 8. Kernel Density function fitted to the data of the parameter 'intensity | fullness'.

In the next step, an appropriate Copula function to estimate the joint probabilities based on the marginal distributions has to be identified. The theory of Copulas is based on Sklar's theorem, stating that for any given joint distribution $\mathrm{F}_{\mathrm{x}, \mathrm{y}}(\mathrm{x}, \mathrm{y})$ with the marginal distributions $\mathrm{F}_{\mathrm{x}}(\mathrm{x})$ and $\mathrm{F}_{\mathrm{y}}(\mathrm{y})$ a Copula $\mathrm{C}$ exists, which links the marginal distributions to the joint distribution:

$$
F_{x, y}(x, y)=C\left[F_{x}(x), F_{y}(Y)\right]
$$

Similar to the univariate parametric distribution functions listed in Table 1, many different types of Copulas (known as Copula families) exist. In the hydrology sector mostly Archimedean Copulas are applied, as they are easy to construct and able to capture asymmetries in the structure of dependence overlooking the margins. Archimedean Copulas are one parametric functions depending on the parameter $\theta$ and they are constructed based on so called Copula generators $\varphi(t)$ :

$$
C_{\theta}(u, v)=\varphi^{-1}[\varphi(u)+\varphi(v)] \text {, with } u=f_{x}(x) ; v=f_{y}(y)
$$

Table 2 gives an overview of the Archimedean Copula functions, which are considered in the present study and widely used in the hydrology sector. For more information on the Copula theory, the mathematical background and potential application cases, the interested reader is referred to Nelsen (1999). 
Table 2. Overview of the 2-dimensional Archimedean Copulas considered in the present study.

\begin{tabular}{|c|c|c|c|}
\hline $\begin{array}{l}\text { Copula-Function } \\
\qquad \mathrm{C}_{\theta}\end{array}$ & $\begin{array}{c}\text { Generator } \\
\varphi(t)^{* *}\end{array}$ & $\begin{array}{c}\text { Range } \\
\Theta\end{array}$ & $\begin{array}{c}\text { Kendall's tau } \\
\mathrm{T}\end{array}$ \\
\hline \multicolumn{4}{|l|}{ Clayton (or Cook-Johnson) } \\
\hline$\left[u^{-\theta}+v^{-\theta}-1\right]^{-1}$ & $\mathrm{t}^{-\theta}-1$ & {$[0, \infty)$} & $\frac{\theta}{\theta+2}$ \\
\hline \multicolumn{4}{|l|}{ Frank } \\
\hline$-\frac{1}{\theta} \ln \left[1+\frac{\left(e^{-\theta u}-1\right)\left(e^{-\theta v}-1\right)}{e^{-\theta}-1}\right.$ & $-\ln \left(\frac{e^{-\theta t}-1}{e^{-\theta}-1}\right)$ & $(-\infty, \infty) \backslash\{0\}$ & $1-\frac{4}{\theta}\left[1-D_{1}(\theta)\right]$ \\
\hline \multicolumn{4}{|l|}{ Gumbel (or Gumbel-Hougaard) } \\
\hline $\exp \left\{-\left[(-\ln u)^{\theta}+(-\ln v)^{\theta}\right]^{\frac{1}{\theta}}\right.$ & $(-\ln t)^{\theta}$ & {$[1, \infty)$} & $1-\theta^{-1}$ \\
\hline * 1. Debye-Function $D_{1}(\theta)=\frac{1}{\theta}$ & $\frac{t}{-1} d t$ & & \\
\hline${ }^{* *} \mathrm{t}=\mathrm{u}$ or $\mathrm{t}=\mathrm{v}$ & & & \\
\hline
\end{tabular}

Analogous to the application of univariate distribution functions, goodness of fit tests are used in the multivariate case to identify the most suitable Copula function to statistically analyze the underlying data set. Here, we apply a graphical based goodness of fit test to identify the Copula which is most qualified to model the structure of dependence between the two storm surge parameters 'highest turning point' and 'intensity | fullness'. Therefore, the two parameters calculated from 100,000 of the stochastically simulated storm surges (including the observations) are compared to $1,000,000$ pairs of the two parameters simulated via the different theoretical Copula functions. The latter are re-transformed into the original units through the marginal distributions.

As it can be seen from Figure 9, only the Gumbel Copula is able to model the structure of dependence between the two storm surge parameters. Therefore it is used to estimate the joint probabilities of the parameters or the occurrence probabilities of the observed and simulated storm surge events, respectively. Figure 10 shows the results from the statistical assessment with some relevant joint exceedance probabilities displayed in the plot. Therewith it is possible to estimate the occurrence probability of any storm surge event, either resulting from numerical simulations, empirical studies or from stochastic simulations.

It has already been mentioned above, that the stochastically simulated storm surge curves can also directly be considered within the next steps of the risk analyses in addition to the results from the empirical studies and the numerical simulations. Following this approach it is possible to consider a very large number of storm surges for the risk analyses instead of only a few storm surges, as the empirical construction of an extreme storm surge event is very time consuming and the number of numerical model runs is restricted due to the computational requirements.

The minimum height of the flood protection for Hörnum was found to be $420 \mathrm{cmNN}$ in the harbor area. This means that only storm surge events with a maximum water level $>420 \mathrm{cmNN}$ are relevant to be considered in a risk analyses, where the risk is defined as the product of the failure probability and the potential losses. Figure 10 shows that none of the observed events exceeded a water level of 420 cmNN, whereas a large number $(>3500)$ of stochastically simulated storm surges is available from $1,000,000$ simulation runs to potentially be analyzed within the risk analyses. 


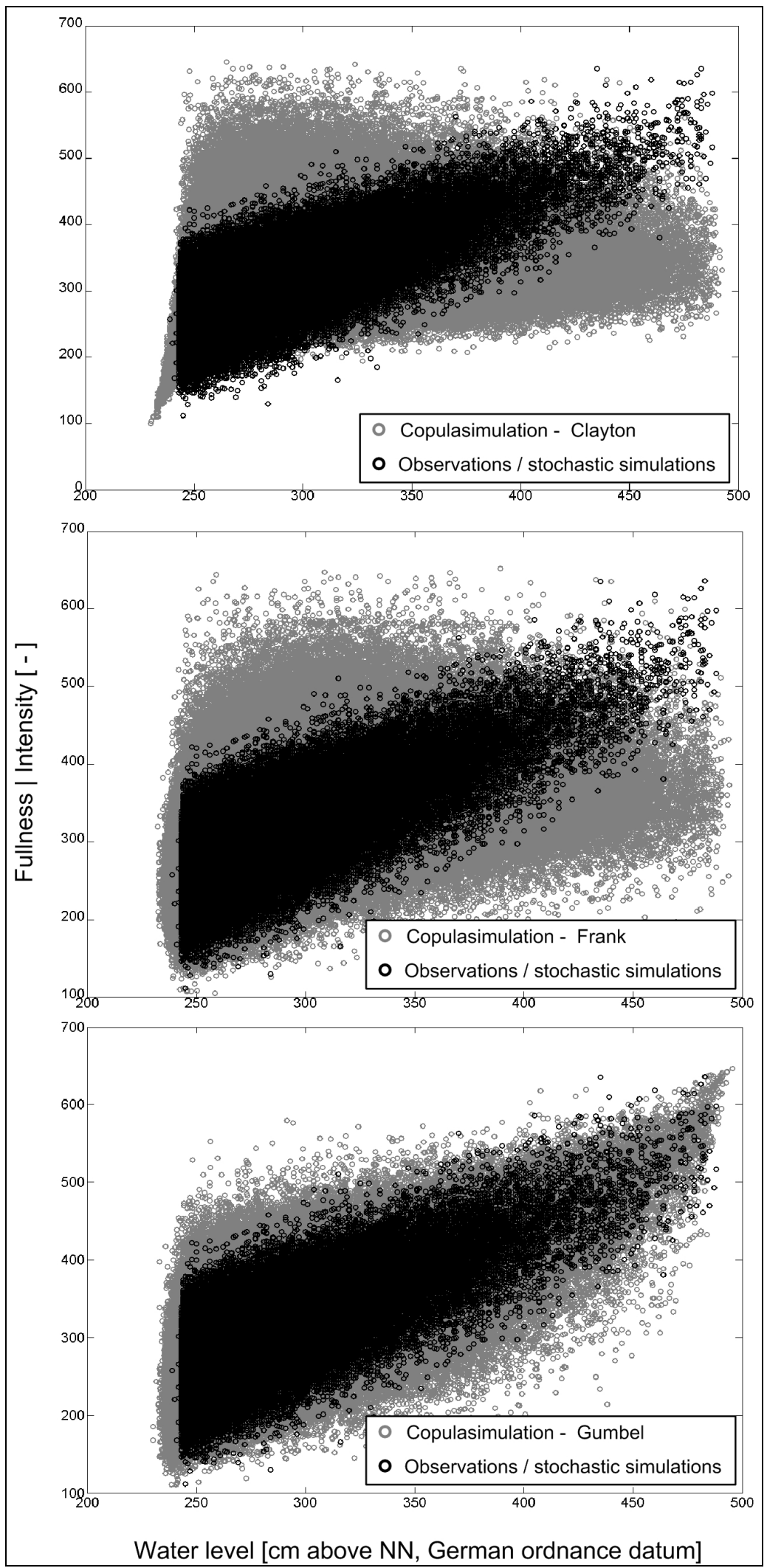

Figure 9. Results from comparing the parameters 'highest turning point' and 'intensity | fullness' from 100,000 stochastically simulated storm surge curves and 1,000,000 simulated pairs of the same parameters based on different theoretical Copula functions (top: Clayton, middle: Frank, bottom: Gumbel). 


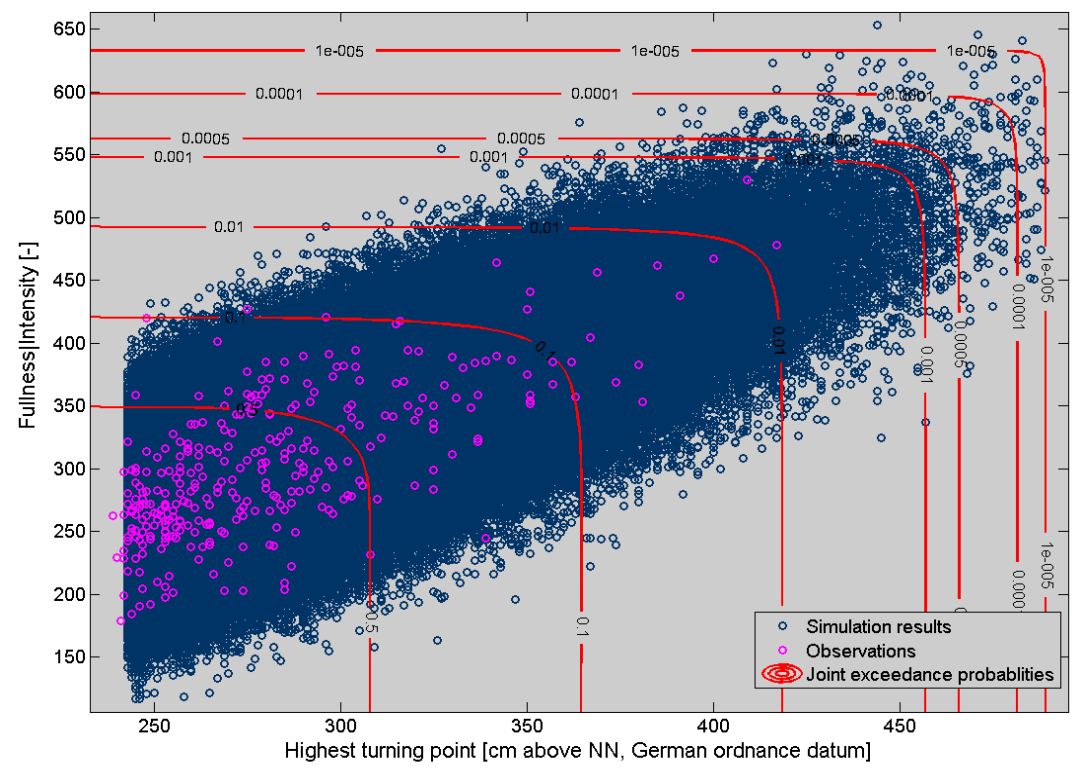

Figure 10. Observed and simulated storm surge events and relevant joint exceedance probablities of the parameters 'highest turning point' and 'intensity | fullness' estimated based on a Gumbel Copula

\section{CONCLUSIONS AND OUTLINE}

In this paper, the applied methodology to statistically analyze extreme storm surge events in the south-eastern part of the North Sea is presented. The results serve as input data for integrated risk analyses for two selected investigation areas (Sylt and Hamburg). A stochastic storm surge generator allowing the simulation of a large number of synthetic and physically consistent storm surge events is developed. Results from numerical model studies on extreme events in the German Bight are used as boundary conditions. For the statistical assessment, an advanced approach based on 2-dimensional Copula functions is applied. For the first time not only the highest water levels during storm surge events are considered, but also the storm surge intensities, which is a decisive parameter (in addition to the water level height) when modeling the failure mechanisms of flood protection measures and estimating potential losses in the hinterland. Thus, it is a parameter which is valuable to be included in the statistical assessment. Based on the results of the stochastic storm surge simulation and the multivariate statistics it is possible to estimate occurrence probabilities for extreme storm surge events, whereas the complete storm surge curves are implicitly addressed.

In addition, the stochastically simulated storm surge curves can be considered for the risk analyses, which, in combination with the consideration of the results from empirical and numerical model studies, reduces the uncertainties and improves the overall results.

At the moment wave conditions are not taken into account. But, in the next step of the XtremRisKproject it is deliberated to extend the available models to additionally consider the wave conditions within the statistical analyses.

\section{ACKNOWLEDGEMENTS}

The joint project XtremRisK is funded by the German Federal Ministry of Education and Research (BMBF). Moreover, the collaboration and the provision of data by the Cooperative and Consulting Partners of the project such as LKN Husum (Schleswig-Holstein Agency for Coastal Defence, National Park und Marine Conservation), HPA Hamburg (Hamburg Port Authority) and LSBG Hamburg (Agency of Roads, Bridges and Water, Hamburg) are gratefully acknowledged.

\section{REFERENCES}

Bowman, A. W. and A. Azzalini. 1997. Applied Smoothing Techniques for Data Analysis, Oxford University Press.

De Michele, C., G. Salvadori, G. Passoni and R. Vezzoli. 2007. A multivariate model of sea storms using Copulas. Coastal Engineering, 54, 734-751. 
De Waal, D. J. and P.H.A.J.M. van Gelder. 2005. Modelling of extreme wave heights and periods through Copulas. Extremes, 8, 345-356.

Dixon, M.J. and J.A Tawn,. 1995. Extreme sea-levels at the UK A-class sites: optimal site-bysite analysis and spatial analyses for the East Coast. POL Internal Document Number 72.

Dixon, M.J. and J.A. Tawn. 1997. Estimates of extreme sea conditions - final report, spatial analysis for the UK coast. POL Internal Document Number 112.

Gönnert, G., Th. Buß and S. Thumm. 2010. Coastal Protection in Hamburg due to climate change. An example to design an extreme storm surge event. In: Proceedings of the First International Conference "Coastal Zone Management of River Deltas and Low Land Coastlines", Alexandria, Egypt.

Gumbel, E.J. 1962. Multivariate extremal distributions. Bull. Inst. Internat. Statist. 39 , pp. 469-475 Paris.

Jensen, J., C. Mudersbach, S. Müller-Navarra, I. Bork, C. Koziar and V. Renner. 2006. Modellgestützte Untersuchungen zu Sturmfluten mit sehr geringen Eintrittswahrscheinlichkeiten an der Deutschen Nordseeküste. Die Küste, 71.

Kahaner, D., C. Moler and S. Nash. 1989. Numerical Methods and Software, Prentice-Hall, Series In Computational Mathematics, ISBN:0-13-627258-4.

Karmakar, S. and S.P. Simonovic. 2008. Bivariate flood frequency analysis: Part 1 - Determination of marginals by parametric and nonparametric techniques. Journal of Flood Risk Management, 1, 190-200.

Karmakar, S. and S.P. Simonovic. 2009. Bivariate flood frequency analysis: Part 2 - A Copula-based approach with mixed marginal distributions. Journal of Flood Risk Management, 2-1, 32-44 (13).

Klein, B., M. Pahlow, Y. Hundecha, C. Gattke and A. Schumann. 2008. Probabilistic Analysis of Hydrological Loads to Optimize the Design of Flood Control Systems. 4th International Symposium on Flood Defence: Managing Flood Risk, Reliability and Vulnerability, Toronto, Canada.

Nelsen, R. B. 1999. An introduction to Copulas. Lecture Notes in Statistics, 139, Springer, New York.

Oumeraci, H. 2004. Sustainable coastal flood defences: scientific and modelling challenges towards an integrated risk-based design concept. Proc. First IMA International Conference on Flood Risk Assessment, IMA - Institute of Mathematics and its Applications, Session 1, Bath, UK, pp. 9-24.

Oumeraci, H., J. Jensen, G. Gönnert, E. Pasche, A. Kortenhaus, M. Naulin, T. Wahl, S. Thumm, G. Ujeyl, I. Gershovich, and A. Burzel. 2009. Flood Risk Analysis for a Megacity: The German XtremRisK-Project, European and Global Communities combine forces on Flood Resilient Cities, Paris, France.

Tawn, J.A.. 1988. Bivariate extreme value theory: models and estimation. Biometrika 75, 397-415.

Tawn, J.A.. 1992. Estimating probabilities of extreme sea-levels. Applied Statistics 41, 77-93. 